bekräftigte damit die Forderung der DFG nach besserer Infrastruktur auf diesem Gebiet.

Mit Hilfe der Spin-Bewegungen von Atomen im starken Magnetfeld könnten, so Leibfritz, in der Pädiatrie die Überlebenschancen von Neugeborenen mit Cerebralschäden prognostiziert werden. Anstelle der invasiven Nadel-Biopsie kann die In-vivo-NMR-Spektroskopie ischämische Schäden in der Myopathie feststellen. Charakterisierung und Therapie von Tumoren in schwer zugänglichen Gebieten (Gehirn) sind möglich. In der Leber sind z.B. Fructose-Intoleranz oder dauernde Alkoholschäden zu identifizieren. Die Aufzeichnung des Energiestoffwechsels ermöglicht die Vorhersage von Herzinfarkten; man kann z.B. die Wirkung des Calcium-Antagonisten Nifedipin feststellen.

Wichtig ist für Leibfritz, daß jetzt schnelle Bildgebungsverfahren (200 ms pro Bild) entwickelt werden. Damit sind, wie er in einem Videofilm zeigte, Bewegungen des schlagenden Herzens oder Schluckbewegungen sichtbar zu machen.

Eines der größten Probleme sieht die DFG in der Nachwuchspflege: Sie wurde in den letzten Jahren sträflich vernachlässigt. Es gebe sicherlich mehr als zwölf Arbeitsgruppen vor allem in der Naturstoff- und Biochemie in der Bundesrepublik, die im Prinzip für ihre Arbeiten ein $600-\mathrm{MHz}$ Gerät benötigen. Der Geräteausschuß der DFG resümiert jedoch: „Leider gibt es aber derzeit deutlich weniger als zwölf Arbeitsgruppen, deren Mitglieder als so qualifiziert gelten können, daß die Beschaffung eines so aufwendigen Geräts gerechtfertigt erscheint.“

\section{Bundesforschungsministerium will astronomische Hochschulgruppen unterstützen}

Von der Idee einer Mars-Reise zeigte sich Bundesforschungsminister Riesenhuber sichtlich hingerissen. In einer Pressekonferenz zur Situation von Astronomie und Astrophysik am 11. August in Bonn meinte er aber, eine solche Planetenreise werde mit mehr als $100 \mathrm{Mrd}$ DM sehr teuer und könne nur gemeinsam mit den USA, der Sowjetunion und der Europäischen Raumfahrtagentur (ESA) verwirklicht werden.

Was der Bund in der sonstigen Planeten- und Sterneforschung tun kann, nimmt sich dagegen weitaus bescheidener aus. Er kommt zwar mit etwa 220 Mio DM jährlich vor allem für Großgeräte - irdische Teleskope und Beobachtungsgeräte im Weltraum - auf, und etwa halb so viel steuern die Länder für Astronomie bei. Woran es aber fehlt, ist die Infrastruktur für Grundlagenforschung in den kleineren Instituten. Zur Zeit arbeiten an 27 Einrichtungen rund 700 Wissenschaftler und Techniker auf dem Gebiet der Astronomie und Astrophysik.

Vor allem versucht das Bundesforschungsministerium, einige der ärgsten Mißstände zu beheben, die die Deutsche Forschungsgemeinschaft in ihrer Astronomie-Denkschrift (Naturwissenschaften aktuell 9/87) dargelegt hat. Vom nächsten Jahr an sollen Hochschulgruppen unterstützt werden, die in einem wellenlängenübergreifenden Verbund Beobachtungsdaten von mehreren Observatorien auswerten. Sie müssen dazu mit einem Rechnernetz verbunden sein. Dieser
Teil eines Deutschen Astronetzes soll im Rahmen des Deutschen Forschungsnetzes ebenfalls vom BMFT finanziert werden. Voraussetzung dazu ist allerdings, daß die Bundesländer an den Hochschulinstituten die entsprechenden Bildverarbeitungsgeräte fördern.

\section{Expertengremium für soziale und ethische Aspekte der Genforschung}

Ein Gremium von 20 Experten, das sich mit den ethischen und sozialen Aspekten bei der Erforschung der menschlichen Erbanlagen befaßt, wurde am 8. September in Bonn eingerichtet. Bis zum Sommer 1990 soll es eine Empfehlung für die Regierung ausarbeiten, wie weit diese Forschung und ihre Förderung bei der Sequenzierung des Genoms sowie bei der Anwendung ihrer Erkenntnisse in Familienberatung, pränataler Diagnostik, Arbeits- und Rechtsleben gehen können. Die Mitglieder des interdisziplinären Arbeitskreises wurden von Bundesforschungsminister Riesenhuber aus Wissenschaftsorganisationen, Berufsverbänden und anderen Einrichtungen berufen. Ihr Vorsitzender ist der Bonner emeritierte Professor Dr. Franz Böckle.

Er hat reichlich Erfahrung in der Sache, um die es geht: Wie nimmt die Gesellschaft die gentechnischen Manipulationen am Menschen selbst auf? Seit Jahren plagen sich regierungsamtliche Kommissionen, eine parlamentarische EnqueteKommission, viele Tagungen und Bücher mit diesen Fragen. Als einer der wenigen Geisteswissenschaftler, die sich mühsam in dieses Gebiet eingearbeitet haben und den Naturwissenschaftlern sagen können, wo sie die Grenzen ihres Könnens sehen müssen, ist der katholische Moraltheologe nun Vorsitzender des neuen Arbeitskreises, der sich mit ethischen und sozialen Folgen der Genforschung am Menschen befaßt.

Vor wenigen Jahren noch war das ein eher nebensächliches Thema. Die von Justiz- und Forschungsminister 1984 eingesetzte Benda-Kommission konzentrierte sich auf Fortpflanzungsmedizin und künstliche Befruchtung; dazu liegt jetzt auch ein streng auf das Strafrecht beschränkter Gesetzentwurf der Bundesregierung zum Embryonenschutz vor (Naturwissenschaften aktuell 8/89). Ein anderes Gesetz soll die industrielle Anwendung der Gentechnik regeln. Beide wurden am 22. September im Bundesrat behandelt, teils an die Regierung zurückgegeben und mit vielen Änderungswünschen befrachtet.

Rechtlich und ethisch noch völlig ungesichert ist, was die Analyse der gesamten menschlichen Erbanlage, des Genoms, bescheren kann. Da sind so wichtige Gebiete wie die vorgeburtliche Diagnostik möglicher Erbkrankheiten, die Identifizierung von künftigen Krankheiten bei Arbeitnehmern - die dann für bestimmte Tätigkeiten nicht in Frage kämen -, Risikoeinschätzungen von Versicherungen, der genetische Fingerabdruck als Beweismittel im Strafverfahren. Juristische Fragen dazu bearbeitet derzeit eine Arbeitsgruppe von Bund und Ländern.

Genomanalyse ist weltweit ein attraktives Thema der Grundlagenforschung geworden. Mit Milliardenbeträgen entschlüsseln die Wissenschaftler weltweit den genetischen Code. Das heißt: In den Genen - der Mensch besitzt 50000 